\title{
In-Service Fatigue Fracture Mechanisms in Covered Disks of a TV3-117VK Helicopter Turbine Engine
}

\author{
A. A. Shanyavskii ${ }^{1, a}$ and Yu. A. Potapenko ${ }^{1}$ \\ ${ }^{1}$ State Centre for Civil Aviation Flight Safety, Sheremetievo-1, Moscow Region, Russia \\ ${ }^{a}$ shana@flysafety.msk.ru
}

In-service propagating fatigue cracks were examined in EI-698VD superalloy thin turbine covered disks of the first, second, and third stages of a TV3-117VK helicopter engine within service lufes of 200-1800 h. To reveal causes of early crack initiation and estimate a propagation time, metallographic and fractographic analyses were performed. The sequence of events during fatigue cracking of the disks was established. A block of striations on the fracture surface was discovered, which characterized fatigue crack propagation during one flight of a helicopter under different operating conditions. The number of striations in each block varied over the range of 4-20, being much more than those used to design covered disks. Fractographic results were used to estimate fatigue crack growth in covered disks of different stages using data on different operating conditions of helicopters.

Keywords: fatigue fracture, low-cycle fatigue, turbine, superalloy, striations, damage mechanisms, aircraft engine, number of flights.

Introduction. In-flight accident of a KA-32 helicopter happened during wood transportation in Malaysia [1]. The collapse of this helicopter was due to in-flight failure of a TV3-117VK turbine engine [1]. The time in service of this engine was $1698 \mathbf{~ h}$ by the moment of the accident, including $674 \mathrm{~h}$ after the last repair. The transportation time was 30-55 $\mathrm{min}$.

The examination of the engine revealed a hole on the top of its right part caused by the body fracture (Fig. 1). The fracture zone was located in the vicinity of II covered disk (CD) of the turbine compressor (TC) [2].

The examination of the engine revealed the failed CDs of the II and III stages of the TC. Both CDs were installed during the last repair. Their time in service was $674 \mathrm{~h}$. The $\mathrm{CD}$ of the first stage was not replaced during the last repair, its operating time was $1710 \mathrm{~h}$.

The results of investigation of fracture surfaces in the failed CDs of the I-III stages of a collapsed KA-32 helicopter engine and other CDs of TV3-117VK engines (500-1500 flight hours) are reviewed below. The in-flight fatigue fracture mechanism of the CDs is briefly discussed.

Research Procedure. The in-service cracked CDs of the TC II and III stages of a TV3-117 engine were statistically analyzed. The analysis has shown that crack initiation in CDs occurred near the hole $3.6 \mathrm{~mm}$ in diameter (Fig. 1), used to position CDs. Cracks propagating from the hole in the II CD appeared after about $300 \mathrm{~h}$ (Fig. 1c). Cracks were regularly observed after in-service time of more than $400 \mathrm{~h}$. The number of cracked (not failed) disks increased with time. First cases of III CDs cracking were registered within $600-800 \mathrm{~h}$. The replacement of the III CDs during repair demonstrates the absence of correlation between the number of cracks and time in service.

Several in-service cracked CDs of the I-III stages of TV3-117VK engines were chosen for analysis at the moment of their repair after $500-1500 \mathrm{~h}$ in service.

All CDs were manufactured from a EI-698VD superalloy. Its chemical composition, mechanical properties and the $\gamma^{\prime}$-phase structure were consistent with recommendations for a EI-698VD superalloy. The composition of the material (in wt.\%) is given in Table 1. 
$\mathrm{T}$ a b 1 e 1

Recommended and Actual Material Composition of an Alloy for In-Service Failed CDs

\begin{tabular}{|c|c|c|c|c|c|c|c|}
\hline $\begin{array}{c}\text { Composition } \\
\text { of a EI-698VD alloy }\end{array}$ & $\mathrm{Ni}$ & $\mathrm{Si}$ & $\mathrm{Mn}$ & $\mathrm{Cr}$ & $\mathrm{Ti}$ & $\mathrm{Mo}$ & $\mathrm{Nb}$ \\
\hline Recommended & $\begin{array}{c}\text { Residual } \\
\text { content }\end{array}$ & $<0.6$ & $<0.4$ & $13.0-16.0$ & $2.35-2.75$ & $2.80-3.20$ & $1.8-2.2$ \\
\hline II CD & ditto & $<0.6$ & $<0.4$ & 15.7 & 2.57 & 2.93 & 2.1 \\
\hline III CD & ditto & $<0.6$ & $<0.4$ & 15.3 & 2.60 & 3.07 & 1.9 \\
\hline
\end{tabular}

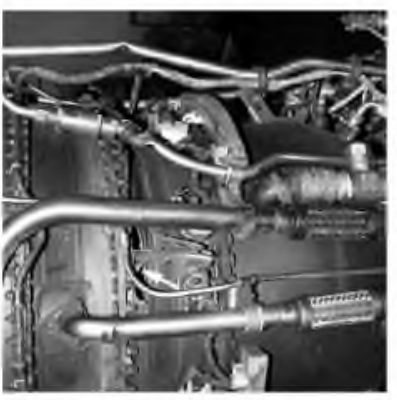

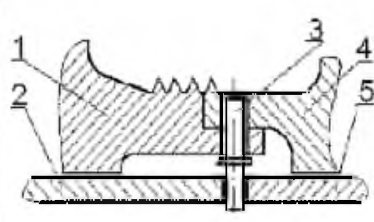

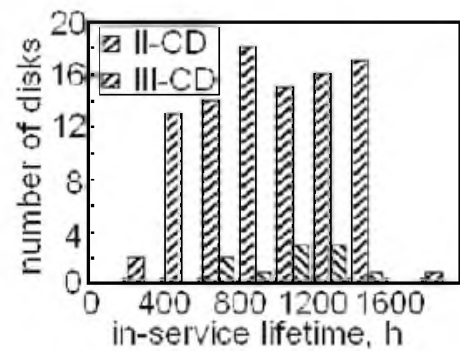

Fig. 1. Overview of a TV3-117VK engine area with the failed zone (a); scheme of the TK of a TV3-117 engine in the vicinity of a hole (b) with pin (3) of the II (I) and III (4) CDs of the II (2) and III (5) TC stages, and the number of cracked disks vs. in-service lifetime for the II and III CDs (c).

Pieces with detected cracks were cut out from the disks, and the fracture surfaces were prepared for fractographic analysis on a Zeiss scanning electron microscope $(3 \mathrm{~nm}$ resolution).

Research Results for CDs of a In-Flight Collapsed Engine. The five fragments are representative of the whole range of fracture patterns in the II CD. The analysis of fracture surfaces demonstrates that fatigue fracture of the II CD mainly occurred in fragments 2 and 3. Crack propagation at the first stage was directed outward from the hole, then the crack was growing along the II CD hub (inward). Intensive oxidation of the fracture surface happened in the inward direction at a distance up to $15 \mathrm{~mm}$.

Fractographic analysis of the II CD fragments revealed that:

(i) crack propagation in the outward direction initiated from one or several origins located near the hole (Fig. 1);

(ii) on the fracture surface, not far from an origin, striations were observed and they represented the block of finer striations within 4-20 (Fig. 2);

(iii) fatigue cracks grew in the outward direction but did not reach the edge of a disk, when the mechanism of the crack growth by the striation formation changed to the intergranular mechanism of static or quasistatic fracture;

(iv) crack growth in the inward direction was controlled by the mechanism of the striation formation down to a depth of about $1.5 \mathrm{~mm}$; then the area of striations in the crack growth direction was gradually reduced, but intergranular cracking was activated, dominating at a crack depth of more than $3 \mathrm{~mm}$.

Fractographic analysis of the I and III CDs demonstrated that:

(v) crack propagation in the I CDs occurred from several holes, and blocks of fatigue striations, detected on the fracture surfaces, were similar to those in the II CD (Fig. 2);

(vi) the III CDs displayed only one zone of fatigue fracture from one of the holes with striation formation in the outward direction. 


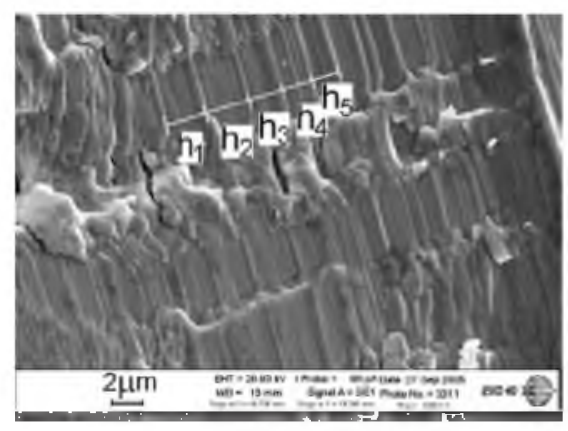

a

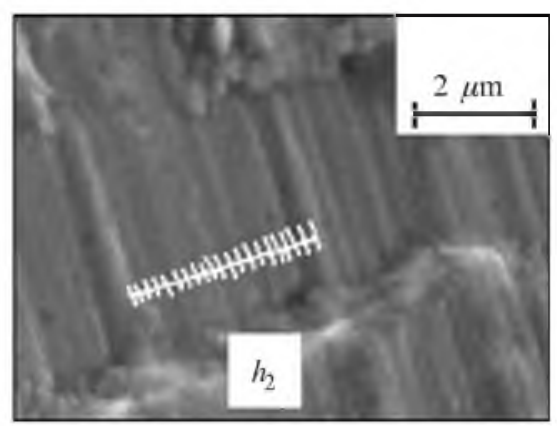

b

Fig. 2. Blocks (a) of striations $h_{i}$ in fatigue fracture surface of the I-CD of the in-flight failed engine and (b) 21 striations in one of these blocks.

Fractographic analysis showed similar fracture behavior of CDs of different stages.

Thus, CD fatigue cracking occurred in a low-cycle fatigue range. The difference in the number of striations in each block reflects the difference in the number of events determined by operating conditions of each flight. This problem was discussed earlier [2]. The same pattern was observed on a strip-chart, which registered in-flight power variations during wood transportation. This fragment, represented a sequence of 21 cycles of power variations as a result of helicopter height variations.

Therefore, each block of striations was considered as a crack increment during one flight. Based on this correlation, fatigue crack growth was estimated in terms of the number of flights for all CDs (Fig. 3).

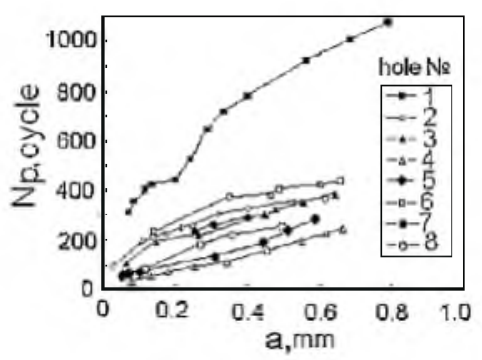

a

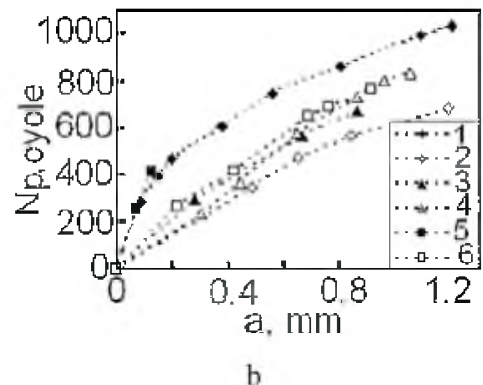

b

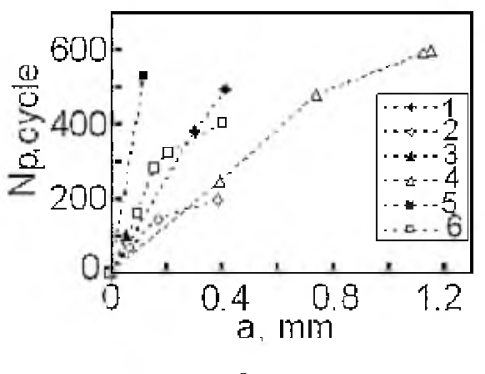

Fig. 3. Number of flights, $N_{p}$, vs. crack length, $a$, for the I-CD in the inward direction (a) and for the II-CD after $1057 \mathrm{~h}$ in the outward (b) and inward (c) directions. Holes are indicated by numbers.

The examination of disks after different times in service allowed the following conclusions: 
1) cracks in II CDs originate after minimum 180 flights and their propagation in both directions from any hole occurs as follows: a crack initiates and grows in the outward direction, and further after certain delay, a crack propagates inward;

2) in the II CDs, the onset of crack propagation inward occurred after a crack propagating outward after minimum 170 flights.

Thus, the II-CDs are the first where the fatigue cracks originate after the service time not less than 180 flights. Then cracks initiate from holes in the outward direction and after propagation during minimum 170 flights, they initiate in the inward direction and grow to the catastrophic fracture.

Discussion. Analysis of CD fracture surfaces gave the following results for disks:

1. The II CD exhibits fatigue cracking with monotonous dependence of striation spacing on the crack length in both directions from the hole; growth period for the main crack was about 600 flights outward and 700 flights inward; transition to the mechanism of intergranular fracture at a crack length of $1.5 \mathrm{~mm}$ occurred after 700 flights.

2. The III CD fatigue cracking took place in the outward direction from the hole during 580 flights.

3 . In the I CD, from one of the holes crack propagates more intensively during about 1100 flights, whereas from other holes a crack growth period did not exceed 400 flights.

Crack growth for the II CD was estimated within crack lengths from $1.5 \mathrm{~mm}$ to $15 \mathrm{~mm}$, where intergranular fracture was dominant. The discovered dependences of the number of flights on the crack length for cracks propagating from two holes to a length of $1.5 \mathrm{~mm}$ were mathematically approximated. Then these dependences were used to calculate the number of flights within crack lengths of $1.5-15 \mathrm{~mm}$. The approximations showed that the crack growth within $1.5-15 \mathrm{~mm}$ took place during $420-650$ flights.

The minimum duration of the main crack growth to the moment of the catastrophic fracture of the II CD was $450+420=870$ flights. The total time in service of the disk includes periods of crack initiation and propagation in two directions and is the sum $180+170+870=1220$ flights.

Thus, fatigue cracks in the I and II CDs took place after the last repair, but were not detected. The change of loading conditions for the I CD at the crack depth of about $0.2 \mathrm{~mm}$ was due to its rearrangement during repair (see Fig. 3a, hole No. 1).

It is recommended to improve non-destructive testing of CDs during their repair to reveal cracks of a length of $0.1-0.3 \mathrm{~mm}$ in depth that could not be detected on the disk side-surface.

1. A. A. Shanyavskiy and Yu. A. Potapenko, Science Works SIAA, 18, 51 (2006).

2. I. A. Birger and B. F. Balashov, Strength of Structural Materials and Components of Aircraft Engines [in Russian], Mashinostroenie, Moscow (1981).

3. A. A. Shanyavskiy, Tolerable Fatigue Cracking of the Aircraft Components. Synergy in Engineering Applications [in Russian], Monograph, Ufa (2003). 